\title{
A Note on Determinant and Adjoint of Fuzzy Square Matrix
}

\author{
Mamoni Dhar \\ Ass istant Professor, Science College, Kokrajhar-783370, Assam, India \\ E-mail: mamonidhar@rediffmail.com
}

\begin{abstract}
The main intention of this article is to represent fuzzy matrices with the help of reference function.Thereafter addition and multiplication of fuzzy matrices are defined keeping in pace with the newly represented fuzzy matrices. Here we study the determinant theory as well as the adjoint theory of square fuzzy matrices. The contribution of th is article is to put forward a new way of expanding the determinant of fuzzy matrices and this process has led the foundation for defining the adjoint of square fuzzy matrices in a quite different way. In the process some properties of determinant as well as adjoint of fuzzy matrices are considered which are found to be almost analogus with the properties in crisp cases.
\end{abstract}

Index Terms - Members hip Value, Reference Function, Boolean Matrices

\section{Introduction}

Matrices with entries in $[0,1]$ and matrix operation defined by fuzzy logical operations are called fuzzy matrices. All fuzzy matrices are matrices but every matrix is not a fuzzy matrix. Fuzzy matrices play a fundamental role in fuzzy set theory. They provide us with a rich framework within which many problems of practical applications of the theory can be formulated. Fuzzy matrices can be successfully used when fuzzy uncertainty occurs in a problem. These results are extensively used for cluster analysis and classification problem of static patterns under subjective measure of similarity. On the other hand, fuzzy matrices are generalized Boolean matrices which have been studied for fruitful results. And the theory of Boolean matrices can be back to the theory of matrices with non negative contents, for which most famous classical results were obtained 1907 to 1912 by Parren and Frobenius. So the theory of fuzzy matrices is interesting in its own right. An important connection between fuzzy sets and fuzzy matrices has been recognized and this has led us to define fuzzy matrices in a quite different way.

Fuzzy matrix has been proposed to represent fuzzy relation in a system based on fuzzy set theory [1]. Fu zzy matrices were introduced first time by Thomson [2], who discussed the convergence of powers of fuzzy matrices. Several authors had presented a number of results on the covergence of power sequences of fuzzy matrices. Several authors have presented a number of results on the convergence of power sequence of fuzzy matrices [3, 4, 5]. Fuzzy matrices play an important role inscience and technology. Kim [6,7] represented some important result on the determinant of a square matrix. He defined the determinant of a square fuzzy matrix and contributed with very research works $[6,7,8,9]$ a lot to the study of determinant theory of square fuzzy matrices. The adjoint of square fuzzy matrix was defined by Thoms on [2] and $\operatorname{Kim}[6]$.

The properties of a square fuzzy matrix are somewhere analogus to the crisp case for determinant of a square fuzzy matrix and also for adjoint of square fuzzy matrices. Since for finding determinant and adjoint of square fuzzy matrices first thing we need is the definition of square fuzzy matrix. Let us have a look at it.

\section{Square Fuzzy Matrices}

A fuzzy matrix is a matrix which has its elements from the interval $[0,1]$, called the unit fuzzy interval. A $m \times n$ fuzzy matrix for which $\mathrm{m}=$ n (i.e the number of rows is equal to the number of columns) and whose elements belong to the unit interval $[0,1]$ is called a fuzzy square matrix of order $n$.

A fuzzy square matrix of order two is expressed in the following way

$$
A=\left(\begin{array}{ll}
a & b \\
c & d
\end{array}\right)
$$

where the entries a,b,c,d all belongs to the interval $[0,1]$.

In accordance with the process of defining complementation of a fuzzy set as defined by Baruah $[10,11,12]$, a fuzzy set

$$
A=\{x, \mu(x), x \in X\}
$$

would be defined in this way as

$$
A=\{x, \mu(x), 0, x \in X\}
$$

so that the complement would become 


$$
A^{c}=\{x, 1, \mu(x), x \in X\}
$$

Thus a square fuzzy matrix $A=\left[a_{i j}\right]_{n \times n}$ would be represented according to the new definition as $A=\left[\left(a_{i j}, 0\right)\right]_{n \times n}$ and similarly the complement matrix of the matrix A would be $A^{c}=\left[\left(1, a_{i j}\right)\right]_{n \times n}$. The following example will make it clear.

The matrix A would be defined according to our way as

$$
A=\left(\begin{array}{ll}
(a, 0) & (b, 0) \\
(c, 0) & (d, 0)
\end{array}\right)
$$

Because we are interested in defining fuzzy sets with the help of reference function.

Similarly the complement of the matrix A would take the form

$$
A^{c}=\left(\begin{array}{ll}
(1, a) & (1, b) \\
(1, c) & (1, d)
\end{array}\right)
$$

It is important to mention here the fact that for finding the adjoint of a square fuzzy matrix, we need the help of determinant of the matrix. It is for this reason; we would like to show the way in which the determinant of a square fuzzy matrix should be expanded in order to keep pace with the representation of fuzzy matrices in terms of reference function. It is seen the representation of fuzzy sets with the help of reference function is very essential in getting logical result which in turn has led the foundation of representing fuzzy matrices with the help of reference function. In the next section, we shall deal with the determinant theory of square fuzzy matrices and some of its properties.

Before proceeding further, let us define two operations which are mostly required in case of finding the determinant of fuzzy matrices

$$
\begin{aligned}
& (\mathrm{a}, \mathrm{b})+(\mathrm{c}, \mathrm{d})=\{\max (\mathrm{a}, \mathrm{c}), \min (\mathrm{b}, \mathrm{d})\} \\
& (\mathrm{a}, \mathrm{b})(\mathrm{c}, \mathrm{d})=\{\min (\mathrm{a}, \mathrm{c}), \max (\mathrm{b}, \mathrm{d})\}
\end{aligned}
$$

The paper is organized as follows: Section II describes the definition of determinant ofsquare fuzzy matrices which is followed by some numerical examples along with the properties of square fuzzy matrices which are discussed in the subsections considered. Section III introduces the new definition of adjoint of square fuzzy matrices which is again followed by some numerical examples and the various properties of square fuzzy matrices are discussed in the subsections of this section. Finally, Section VI presents our conclusions.

\section{Determinant of a Square Fuzzy Matrix}

The determinant of the fuzzy matrix A would be denoted by

$$
|A|=\left|\begin{array}{ll}
(a, 0) & (b, 0) \\
(c, 0) & (d, 0)
\end{array}\right|
$$

and we would like to expand the above determinant in the following way

$$
|A|=\left|\begin{array}{ll}
(a, 0) & (b, 0) \\
(c, 0) & (d, 0)
\end{array}\right|
$$

$=[\max \{\min (\mathrm{a}, \mathrm{d}), \min (\mathrm{b}, \mathrm{c})\}, \min \{\max (0,0), \max (0,0)\}]$

Now for the a square fuzzy matrix of order 3

$$
B=\left(\begin{array}{lll}
\left(a_{1}, 0\right) & \left(b_{1}, 0\right) & \left(c_{1}, 0\right) \\
\left(a_{2}, 0\right) & \left(b_{2}, 0\right) & \left(c_{2}, 0\right) \\
\left(a_{3}, 0\right) & \left(b_{3}, 0\right) & \left(c_{3}, 0\right)
\end{array}\right)
$$

The determinant would be denoted by

$$
\left|\begin{array}{lll}
\left(a_{1}, 0\right) & \left(b_{1}, 0\right) & \left(c_{1}, 0\right) \\
\left(a_{2}, 0\right) & \left(b_{2}, 0\right) & \left(c_{2}, 0\right) \\
\left(a_{3}, 0\right) & \left(b_{3}, 0\right) & \left(c_{3}, 0\right)
\end{array}\right|
$$

and this would be defined as

$$
\begin{aligned}
& =\left(a_{1}, 0\right)\left|\begin{array}{ll}
\left(b_{2}, 0\right) & \left(c_{2}, 0\right) \\
\left(b_{3}, 0\right) & \left(c_{3}, 0\right)
\end{array}\right|+\left(b_{1}, 0\right)\left|\begin{array}{ll}
\left(a_{2}, 0\right) & \left(c_{2}, 0\right) \\
\left(a_{3}, 0\right) & \left(c_{3}, 0\right)
\end{array}\right| \\
& +\left(c_{1}, 0\right)\left|\begin{array}{ll}
\left(a_{2}, 0\right) & \left(b_{2}, 0\right) \\
\left(a_{3}, 0\right) & \left(b_{3}, 0\right)
\end{array}\right|
\end{aligned}
$$

$=\left(a_{1}, 0\right)\left[\max \left\{\min \left(b_{2}, c_{3}\right), \min \left(b_{3}, c_{2}\right)\right\}\right.$, $\min \{\max (0,0), \max (0,0)\}+{ }^{\left(b_{1}, 0\right)}\left[\max \left\{\min \left(a_{2}, c_{3}\right)\right.\right.$ $\left.\min \left(a_{3}, c_{2}\right)\right\}, \quad \min \{\max (0,0), \quad \max (0,0)\}+$

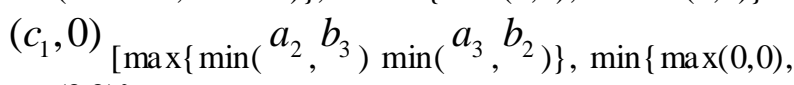
$\max (0,0)\}$.

This is the way of defining the fuzzy matrices in the usual case. But in fuzzy areas there arises some cases when complement of fuzzy matrices is involved. In order to meet the need of the situation it is important to define the complement of fuzzy matrices in our way. We would like to define the complement of fuzzy matrices in accordance with the definition of complement of fuzzy sets as defined by Baruah [10, $11 \& 12]$. This result has been used in the works of Dhar.et.at $[13,14,15,16,17 \& 18]$ to draw some conclusions. The new definition of complementation of fuzzy sets has been discussed in details in our previous 
works and so we would like to mention about in brief in this very article.

It would be easy to proceed with the usual fuzzy matrices whose reference functions are taken as zero and it is due to this reason we would like to focus on the complementation of fuzzy matrices.

Taking into consideration of the above mentioned procedure for representing fuzzy matrices along with the definition of complementation of fuzzy sets, the complement of the above fuzzy matrix B can be written as

$$
\left(\begin{array}{lll}
\left(1, a_{1}\right) & \left(1, a_{2}\right) & \left(1, a_{3}\right) \\
\left(1, b_{1}\right) & \left(1, b_{2}\right) & \left(1, b_{3}\right) \\
\left(1, c_{1}\right) & \left(1, c_{2}\right) & \left(1, c_{3}\right)
\end{array}\right)
$$

Then the determinat of the above complement matrix would be evaluated as

$$
\begin{aligned}
& \left(1, a_{1}\right)\left|\begin{array}{ll}
\left(1, b_{2}\right) & \left(1, c_{2}\right) \\
\left(1, b_{3}\right) & \left(1, c_{3}\right)
\end{array}\right|+\left(1, b_{1}\right)\left|\begin{array}{ll}
\left(1, a_{2}\right) & \left(1, c_{2}\right) \\
\left(1, a_{3}\right) & \left(1, c_{3}\right)
\end{array}\right| \\
& +\left(1, c_{1}\right)\left|\begin{array}{ll}
\left(1, a_{2}\right) & \left(1, b_{2}\right) \\
\left(1, a_{3}\right) & \left(1, b_{3}\right)
\end{array}\right| \\
& =\left(1, a_{1}\right)\left[\max \{\min (1,1), \min (1,1)\}, \min \left\{\max \left(b_{2}, c_{3}\right)\right. \text {, }\right. \\
& \left.\left.\max \left(b_{3}, c_{2}\right)\right\}\right]+\left(1, b_{1}\right)_{[\max \{\min (1,1), \min (1,1)\},}
\end{aligned}
$$

It is to be noted here that the above determinant is expanded along the first row. But it can be easily observed that the value of the determinant remains unchanged if it is expanded along any row or columns.

\subsection{Numerical Examples}

Here we shall put a numerical example to find the determinant of a fuzzy matrix in the way described above.

$$
A^{c}=\left(\begin{array}{ccc}
(1,0.5) & (1,0.3) & (1,0.8) \\
(1,0.6) & (1,0.2) & (1,0.9) \\
(1,0) & (1,0.7) & (1,0.4)
\end{array}\right)
$$

Then the determinant of the above matrix when expanded along the first row would give us the following result

$$
\begin{aligned}
\left|A^{c}\right|= & (1,0.5)\left|\begin{array}{ll}
(1,0.2) & (1,0.7) \\
(1,0.9) & (1,0.4)
\end{array}\right| \\
& +(1,0.6)\left|\begin{array}{ll}
(1,0.3) & (1,0.7) \\
(1,0.8) & (1,0.4)
\end{array}\right| \\
& +(1,0)\left|\begin{array}{ll}
(1,0.3) & (1,0.2) \\
(1,0.8) & (1,0.9)
\end{array}\right| \\
= & (1,0.5)[\max (1,1), \min (0.4,0.9)]+(1,0.3)[\max (1,1), \\
= & \min (0.6,1)]+(1,0.5)[\max (1,1), \min (0.2,0.7)] \\
= & {[\max \{\min (1,0.4)+(1,0.3)\}, \min \{\max (0.5,0.4)\}]+} \\
\max & \{\min (1,1)\}, \min \{\max (0.3,0.6)\}]+[\max \{\min (1,1)\}, \\
\min & \{\max (0.8,0.2)\}] \\
= & (1,0.5)+(1,0.6)+(1,0.8) \\
= & \{\max (1,1), \min (0.5,0.6)\}+(1,0.8) \\
= & (1,0.5)+(1,0.8) \\
= & \{\max (1,1), \min (0.5,0.8)\} \\
= & (1,0.5)
\end{aligned}
$$

\subsection{Some Properties of Determinant of Square Fuzzy Matrix}

This section would deal with some of the properties of the determinant of square fuzzy matrices when the reference function is taken into consideration for representing fuzzy matrices.

\subsubsection{Property 1}

The value of the determinant remains unchanged when any two rows or columns are interchanged.

Let us consider the determinant of the above matrix with $1^{\text {st }}$ and $2^{\text {nd }}$ columns interchanged.

$$
\begin{aligned}
& \left|\begin{array}{ccc}
(1,0.3) & (1,0.5) & (1,0.8) \\
(1,0.2) & (1,0.6) & (1,0.9) \\
(1,0.7) & (1,0) & (1,0.4)
\end{array}\right| \\
= & (1,0.3)\left|\begin{array}{cc}
(1,0.6) & (1,0.9) \\
(1,0) & (1,0.4)
\end{array}\right| \\
+ & (1,0.5)\left|\begin{array}{ll}
(1,0.2) & (1,0.9) \\
(1,0.7) & (1,0.4)
\end{array}\right| \\
+ & (1,0.8)\left|\begin{array}{cc}
(1,0.2) & (1,0.6) \\
(1,0.7) & (1,0)
\end{array}\right|
\end{aligned}
$$

$=(1,0.3)[\max (1,1), \min (0.6,0.9)]+(1,0.5)[\max (1,1)$, $\min (0.4,0.9)]+(1,0.8)[\max (1,1), \min (0.2,0.7)]$

$$
=(1,0.3)(1,0.6)+(1,0.5)(1,0.4)+(1,0.8)(1,0.2)
$$




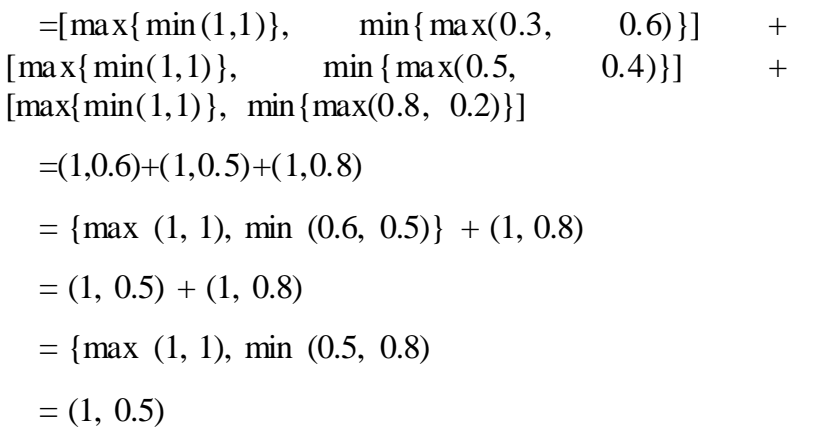

Thus the values of the determinant remain unchanged if the rows and columns are interchanged.

\subsubsection{Propery 2}

The values of the determinant of fuzzy square matrix remain unchanged when rows and columns are interchanged.

Let us consider the following matrix which is obtained by interchanging the rows and columns of the above matrix $A^{c}$

$$
\begin{aligned}
& \left(\begin{array}{lll}
\left(1, a_{1}\right) & \left(1, a_{2}\right) & \left(1, a_{3}\right) \\
\left(1, b_{1}\right) & \left(1, b_{2}\right) & \left(1, b_{3}\right) \\
\left(1, c_{1}\right) & \left(1, c_{2}\right) & \left(1, c_{3}\right)
\end{array}\right) \\
& \left(1, a_{1}\right)\left|\begin{array}{ll}
\left(1, b_{2}\right) & \left(1, b_{3}\right) \\
\left(1, c_{2}\right) & \left(1, c_{3}\right)
\end{array}\right|+\left(1, b_{1}\right)\left|\begin{array}{ll}
\left(1, a_{2}\right) & \left(1, a_{3}\right) \\
\left(1, c_{2}\right) & \left(1, c_{3}\right)
\end{array}\right| \\
& +\left(1, c_{1}\right)\left|\begin{array}{ll}
\left(1, a_{2}\right) & \left(1, a_{3}\right) \\
\left(1, b_{2}\right) & \left(1, b_{3}\right)
\end{array}\right|
\end{aligned}
$$

Then the determinant of the above complement matrix would be evaluated as

$$
\begin{aligned}
& \left(1, a_{1}\right)\left|\begin{array}{ll}
\left(1, b_{2}\right) & \left(1, b_{3}\right) \\
\left(1, c_{2}\right) & \left(1, c_{3}\right)
\end{array}\right|+\left(1, b_{1}\right)\left|\begin{array}{ll}
\left(1, a_{2}\right) & \left(1, a_{3}\right) \\
\left(1, c_{2}\right) & \left(1, c_{3}\right)
\end{array}\right| \\
& +\left(1, c_{1}\right)\left|\begin{array}{ll}
\left(1, a_{2}\right) & \left(1, a_{3}\right) \\
\left(1, b_{2}\right) & \left(1, b_{3}\right)
\end{array}\right|
\end{aligned}
$$

\section{Numerical Example}

With the help of this numerical example, it is expected that the property which is stated to hold true would be clear

Let us consider thefollowing determinant

$$
\left|\begin{array}{ccc}
(1,0.5) & (1,0.6) & (1,0) \\
(1,0.3) & (1,0.2) & (1,0.7) \\
(1,0.8) & (1,0.9) & (1,0.4)
\end{array}\right|
$$

$$
\begin{aligned}
&=(1,0.5)\left|\begin{array}{ll}
(1,0.2) & (1,0.7) \\
(1,0.9) & (1,0.4)
\end{array}\right| \\
&+(1,0.6)\left|\begin{array}{ll}
(1,0.3) & (1,0.7) \\
(1,0.8) & (1,0.4)
\end{array}\right| \\
&+(1,0)\left|\begin{array}{ll}
(1,0.3) & (1,0.2) \\
(1,0.8) & (1,0.9)
\end{array}\right| \\
&=(1,0.5)[\max (1,1), \min (0.4,0.9)]+(1,0.6)[\max (1,1), \\
&\min (0.4,0.8)]+(1,0.8)[\max (1,1), \min (0.9,0.8)] \\
&=(1,0.5)(1,0.4)+(1,0.6)(1,0.4)+(1,0.8)(1,0.8) \\
&=(1,0.5)+(1,0.6)+(1,0.8) \\
&=(1,0.5)+(1,0.8) \\
&=(1,0.5)
\end{aligned}
$$

\subsubsection{Property3}

If $A^{c}$ and $B^{c}$ be two square fuzzy matrices then the following property will hold

$$
\begin{gathered}
\operatorname{det}\left(A^{c} B^{c}\right) \neq \operatorname{det} A^{c} \operatorname{det} B^{c} \\
B^{c}=\left(\begin{array}{ccc}
(1,0.3) & (1,1) & (1,0.7) \\
(1,1) & (1,0.9) & (1,0) \\
(1,0.8) & (1,0.2) & (1,0.3)
\end{array}\right)
\end{gathered}
$$

Proceeding in the similar manner we would get the value of the determinant of $B^{c}$ as $(1,0.9)$.

Hence we get

$$
\begin{aligned}
\operatorname{det}\left(A^{c} B^{c}\right) & =(1,0.5)(1,0.9) \\
& =(1,0.9)
\end{aligned}
$$

Now in order to find $\operatorname{det}\left(A^{c} B^{c}\right)$ we would have to see whether the product of the two fuzzy matrices $A^{c}$ and $B^{c}$ is defined. So we should first define the multiplication of two fuzzy matrices when represented with the help of reference function.

Before proceeding further let us define the multiplication of two fuzzy matrices for illustration purposes.

\subsubsection{Multiplication of Two Square Fuzzy Matrices}

Now after finding addition of fuzzy matrices, we shall try to find the multiplication of two fuzzy matrices .The product of two fuzzy matrices under usual matrix multiplication is not a fuzzy matrix. It is due to this reason; a conformable operation analogus to the product which again happens to be a fuzzy matrix was introduced by many researchers which can be found in 
fuzzy literature which can be found in the reference [1]. However, even for this operation the product $A B$ to be defined if the number of columns of the first fuzzy matrix $\mathrm{A}$ is equal to the number of rows of the second fuzzy matrix B. In the process of finding multiplication of fuzzy matrices, if this condition is satisfied then the multiplication of two fuzzy matrices $A$ and $B$, will be defined and can be represented in the following form:

$$
A B=\left\{\max \min \left(a_{i j}, b_{j i}\right), \min \max \left(r_{i j}, r_{j i}^{\prime}\right)\right\}
$$

$$
\text { Where } A=\left[\left(a_{i j}, r_{i j}\right)\right] \text { and } B=\left[\left(b_{i j}, r_{j i}{ }^{\prime}\right)\right] \text {, }
$$
$1 \leq i \leq n, 1 \leq j \leq n$ and $a_{i j}$, stands for the membership function of the fuzzy matrix $A$ and the corresponding reference function is $r_{i j}$ whereas $b_{i j}$ stands for the membership function of the fuzzy matrix $\mathrm{B}$ with the corresponding reference function ${ }^{{ }^{\prime}{ }^{\prime}}$. Now if we consider two fuzzy square matrices $A^{c}$ and $B^{c}$ as described previously then we have the product $A^{c} B^{c}$ as

$$
\left(\begin{array}{ccc}
(1,0.5) & (1,0.3) & (1,0.8) \\
(1,0.6) & (1,0.2) & (1,0.9) \\
(1,0) & (1,0.7) & (1,0.4)
\end{array}\right)\left(\begin{array}{ccc}
(1,0.3) & (1,1) & (1,0.7) \\
(1,1) & (1,0.9) & (1,0) \\
(1,0.8) & (1,0.2) & (1.0 .3)
\end{array}\right)
$$

Which when calculated with our method of multiplication would give us the following result

$$
=\left(\begin{array}{lll}
(1,0.5) & (1,0.8) & (1,0.3) \\
(1,0.6) & (1,0.9) & (1,0.2) \\
(1,0.3) & (1,0.4) & (1.0 .4)
\end{array}\right)
$$

Now the determinant of the above fuzzy square matrix is $(1,0.5)$ which states the fact that

$$
\operatorname{det}\left(A^{c} B^{c}\right) \neq \operatorname{det} A^{c} \operatorname{det} B^{c}
$$

In the above section, we have gone through the new method of defining the determinant of square fuzzy matrices and thereafter some properties of the determinant are studied. The next section is contributed to deal with the adjoint theory of square fuzzy matrices.

\section{Adjoint of Square Fuzzy Matrix}

Let $A=\left[a_{i j}\right]$ be any matrix. Then $B=\left[b_{i j}\right]_{n \times n}$ where $b_{i j}=\left|A_{i j}\right|$ and $\left|A_{j i}\right|$ is the determinant of the square fuzzy matrix of order (n-1) obtained from a square fuzzy matrix A of order $n$ by deleting row and column $\mathrm{i}$. Then the matrix B is defined as the adjoint of the matrix A. It is to be noted here that the adjoint of a matrix is of the same order as that of the given matrix.

Here in this article, we would like to represent a fuzzy matrix $A=\left[a_{i j}\right]$ in the form after considering reference function to serve our purpose. Then the adjoint will take the form $B=\left[\left(b_{i j}, 0\right)\right]$. The membership value matrix which can be obtained from this representation would coincide with that of usual matrix representation. But before proceeding further let us have a look at the way in which the adjoint of fuzzy matrices are to be defined in our way.

$$
\begin{aligned}
&\left|A^{c}\right|=(1,0.5)\left|\begin{array}{ll}
(1,0.2) & (1,0.7) \\
(1,0.9) & (1,0.4)
\end{array}\right| \\
&+(1,0.6)\left|\begin{array}{ll}
(1,0.3) & (1,0.7) \\
(1,0.8) & (1,0.4)
\end{array}\right| \\
&+(1,0)\left|\begin{array}{ll}
(1,0.3) & (1,0.2) \\
(1,0.8) & (1,0.9)
\end{array}\right| \\
& b_{11}=\left|A_{11}\right|=\left|\begin{array}{ll}
\left(b_{2}, 0\right) & \left(c_{2}, 0\right) \\
\left(b_{3}, 0\right) & \left(c_{3}, 0\right)
\end{array}\right| \\
&=\left[\max \left\{\min \left(b_{2}, c_{3}\right), \min \left(b_{3}, c_{2}\right)\right\}, \min \{\max (0,0), \max \right.
\end{aligned}
$$
$(0,0)\}]$

$$
\begin{aligned}
& b_{12}=\left|A_{21}\right|=\left|\begin{array}{ll}
\left(a_{2}, 0\right) & \left(c_{2}, 0\right) \\
\left(a_{3}, 0\right) & \left(c_{3}, 0\right)
\end{array}\right| \\
& =\left[\max \left\{\min \left(a_{2}, c_{3}\right), \min \left(a_{3}, c_{2}\right)\right\}, \min \{\max (0,0), \mathrm{ma}\right. \\
& \mathrm{x}(0,0)\}] \\
& b_{13}=\left|A_{31}\right|=\left|\begin{array}{ll}
\left(a_{2}, 0\right) & \left(b_{2}, 0\right) \\
\left(a_{3}, 0\right) & \left(b_{3}, 0\right)
\end{array}\right| \\
& =\left[\max \left\{\min \left(a_{2}, b_{3}\right), \min \left(a_{3}, b_{2}\right)\right\}, \min \{\max (0,0), \mathrm{ma}\right. \\
& \mathrm{x}(0,0)\}]
\end{aligned}
$$

Similarly we are to find the others.

Then the adjoint of the matrix B would symbolically be represented as

$$
\operatorname{adj} B=\left(\begin{array}{ccc}
\left(b_{11}, 0\right) & \left(b_{12}, 0\right) & \left(b_{13}, 0\right) \\
\left(b_{21}, 0\right) & \left(b_{22}, 0\right) & \left(b_{23}, 0\right) \\
\left(b_{31}, 0\right) & \left(b_{32}, 0\right) & \left(b_{33}, 0\right)
\end{array}\right)
$$




\subsection{Numerical Example}

To illustrate the introduced concept we want to cite the following numerical example. Let us consider a square fuzzy matrix of order 3 as

$$
A^{c}=\left(\begin{array}{ccc}
(1,0.5) & (1,0.3) & (1,0.8) \\
(1,0.6) & (1,0.2) & (1,0.9) \\
(1,0) & (1,0.7) & (1,0.4)
\end{array}\right)
$$

Then we have

$$
\begin{aligned}
& b_{11}=\left|\begin{array}{ll}
(1,0.2) & (1,0.9) \\
(1,0.7) & (1,0.4)
\end{array}\right| \\
& =[\max \{\min (1,1), \min (1,1) \\
& \min \{\max (0.2,0.4), \max (0.7,0.9)\}] \\
& =[\max (1,1), \min (0.4,0.9)] \\
& =(1,0.4) \\
& b_{12}=\left|\begin{array}{cc}
(1,0.6) & (1,0.9) \\
(1,0) & (1,0.4)
\end{array}\right| \\
& =[\max \{\min (1,1), \min (1,1) \\
& \min \{\max (0.6,0.4), \max (0,0.9)\}] \\
& =[\max (1,1), \min (0.6,0.9)] \\
& =(1,0.6) \\
& b_{13}=\left|\begin{array}{cc}
(1,0.6) & (1,0.2) \\
(1,0) & (1,0.7)
\end{array}\right| \\
& =[\max \{\min (1,1), \min (1,1) \\
& \min \{\max (0.6,0.7), \max (0,0.2)\}] \\
& =[\max (1,1), \min (0.7,0.2)] \\
& =(1,0.2)
\end{aligned}
$$

\section{Similarly}

$$
\begin{aligned}
& b_{21}=(1,0.4), b_{22}=(1,0.5), b_{23}=(1,0.3) \\
& b_{31}=(1,0.8), b_{32}=(1,0.8), b_{33}=(1,0.5)
\end{aligned}
$$

Hence the adjont of the matrix $A^{c}$ will become

$$
\operatorname{adj} A^{c}=\left(\begin{array}{lll}
(1,0.4) & (1,0.6) & (1,0.2) \\
(1,0.4) & (1,0.5) & (1,0.3) \\
(1,0.8) & (1,0.8) & (1,0.5)
\end{array}\right)
$$

\subsection{Property}

\subsubsection{Property1}

If $A^{c}$ be a fuzzy square matrix, then we get

$$
A^{c}\left(\operatorname{adj} A^{c}\right) \neq\left(\operatorname{adj} A^{c}\right) A^{c}
$$

Now let us find the following

$A^{c}\left(\operatorname{adj} A^{c}\right)$

$$
\begin{aligned}
&=\left(\begin{array}{ccc}
(1,0.5) & (1,0.3) & (1,0.8) \\
(1,0.6) & (1,0.2) & (1,0.9) \\
(1,0) & (1,0.7) & (1,0.4)
\end{array}\right) \\
&\left(\begin{array}{lll}
(1,0.4) & (1,0.6) & (1,0.2) \\
(1,0.4) & (1,0.5) & (1,0.3) \\
(1,0.8) & (1,0.8) & (1,0.5)
\end{array}\right) \\
& A^{c}\left(\operatorname{adj}^{c}\right)=\left[C_{i j}\right] \text { (Say) }
\end{aligned}
$$

Where

$=[\max \{\min (1,1), \min (1,1), \min (1,1)\}$, $\min \{\max (0.5,0.6), \max (0.3,0.2), \max (0.8,0.4)\}]$

$=\{\max (1,1$, and 1$), \min (0.6,0.3$, and 0.8$)\}$

$=(1,0.3)$

$=[\max \{\min (1,1), \min (1,1), \min (1,1)\}$, $\min \{\max (0.5,0.4), \max (0.3,0.5), \max (0.8,0.3)\}]$

$=\{\max (1,1$, and 1$), \min (0.5,0.5$, and 0.8$)\}$

$=(1,0.5)$

$=[\max \{\min (1,1), \min (1,1), \min (1,1)\}$, $\min \{\max (0.5,0.8), \max (0.3,0.8), \max (0.8,0.5)\}]$

$=\{\max (1,1$, and 1$), \min (0.8,0.8$, and 0.8$)\}$

$=(1,0.8)$

Similar calculation would give us the following results

$$
\begin{aligned}
& C_{21}=(1,0.2), C_{22}=(1,0.5), C_{23}=(1,0.8) \\
& C_{31}=(1,0.4), C_{32}=(1,0.4), C_{33}=(1,0.5)
\end{aligned}
$$

Thus we see that 


$$
A^{c} \operatorname{adj} A^{c}=\left(\begin{array}{lll}
(1,0.4) & (1,0.5) & (1,0.3) \\
(1,0.4) & (1,0.5) & (1,0.3) \\
(1,0.4) & (1,0.6) & (1,0.2)
\end{array}\right)
$$

Similarly we get

$$
\begin{gathered}
C_{31}=(1,0.4), C_{32}=(1,0.4), C_{33}=(1,0.5) \\
\left(\operatorname{adj} A^{c}\right) A^{c}=\left(\begin{array}{lll}
(1,0.2) & (1,0.4) & (1,0.4) \\
(1,0.3) & (1,0.4) & (1,0.4) \\
(1,0.5) & (1,0.7) & (1,0.5)
\end{array}\right)
\end{gathered}
$$

So here it is observed that in case of fuzzy square matrices

$$
A^{c}\left(\operatorname{adj} A^{c}\right) \neq\left(\operatorname{adj} A^{c}\right) A^{c}
$$

\subsubsection{Property2}

If $A^{c}$ be a fuzzy square matrix, then

$$
\operatorname{det}\left(\operatorname{adj}^{c}\right)=\operatorname{det} A^{c}
$$

We have already found the adjoint of the fuzzy matrix $\operatorname{adj}^{c}$ and so we would like to calculate the determinant of this matrix

$$
\begin{aligned}
&\left|\operatorname{adj}^{c}\right|=(1,0.4)\left|\begin{array}{ll}
(1,0.5) & (1,0.3) \\
(1,0.8) & (1,0.5)
\end{array}\right|+(1,0.6)\left|\begin{array}{ll}
(1,0.4) & (1,0.3) \\
(1,0.8) & (1,0.5)
\end{array}\right| \\
&+(1,0.2)\left|\begin{array}{ll}
(1,0.4) & (1,0.5) \\
(1,0.8) & (1,0.8)
\end{array}\right| \\
&=(1,0.4)[\max \{\min (1,1), \min (1,1)\}, \\
&\quad \min \{\max (0.5,0.5), \max (0.3,0.8)\}] \\
&+(1,0.6)[\max \{\min (1,1), \min (1,1)\}, \\
&\quad \min \{\max (0.4,0.5), \max (0.8,0.3)\}] \\
&+(1,0.2)[\max \{\min (1,1), \min (1,1)\}, \\
&\quad \min \{\max (0.4,0.8), \max (0.5,0.8)\}] \\
&=(1,0.4)(1,0.5)+(1,0.6)(1,0.5)+(1,0.2)(1,0.8) \\
&=(1,0.5)+(1,0.6)+(1,0.8) \\
&=(1,0.5)+(1,0.8) \\
&=(1,0.5) \quad
\end{aligned}
$$

Similarly, we get

$$
\operatorname{det} A^{c}=(1,0.5)
$$

Hence the following result is obtained

$$
\operatorname{det}\left(\operatorname{adj}^{c}\right)=\operatorname{det} A^{c}
$$

\subsubsection{Property3}

If $A^{c}$ be a fuzzy square matrix, we shall show that

$$
\operatorname{det} A^{c}\left(\operatorname{adj} A^{c}\right)=\operatorname{det} A^{c}=\operatorname{det}\left(\operatorname{adj} A^{c}\right) A^{c}
$$

Here

$\operatorname{det} A^{c}\left(\operatorname{adj} A^{c}\right)$

$$
\begin{aligned}
& =(1,0.4)\left|\begin{array}{ll}
(1,0.5) & (1,0.3) \\
(1,0.6) & (1,0.2)
\end{array}\right| \\
& +(1,0.5)\left|\begin{array}{ll}
(1,0.4) & (1,0.3) \\
(1,0.4) & (1,0.2)
\end{array}\right| \\
& +(1,0.3)\left|\begin{array}{ll}
(1,0.4) & (1,0.5) \\
(1,0.4) & (1,0.6)
\end{array}\right|
\end{aligned}
$$

$=(1,0.4)[\max \{\min (1,1), \min (1,1)\}$, $\min \{\max (0.5,0.2), \max (0.3,0.6)\}]$

$+(1,0.5)[\max \{\min (1,1), \min (1,1)\}$, $\min \{\max (0.4,0.2), \max (0.4,0.3)\}]$

$+(1,0.3)[\max \{\min (1,1), \min (1,1)\}$, $\min \{\max (0.4,0.6), \max (0.5,0.4)\}]$

$=(1,0.4)(1,0.5)+(1,0.5)(1,0.4)+(1,0.3)(1,0.5)$

$=(1,0.5)+(1,0.5)+(1,0.5)$

$=(1,0.5)+(1,0.5)$

$=(1,0.5)$

$$
\begin{aligned}
& \operatorname{det}\left(\operatorname{adj} A^{c}\right) A^{c} \\
& =(1,0.2)\left|\begin{array}{ll}
(1,0.4) & (1,0.4) \\
(1,0.7) & (1,0.5)
\end{array}\right| \\
& +(1,0.4)\left|\begin{array}{ll}
(1,0.3) & (1,0.4) \\
(1,0.5) & (1,0.5)
\end{array}\right| \\
& +(1,0.4)\left|\begin{array}{ll}
(1,0.3) & (1,0.4) \\
(1,0.5) & (1,0.7)
\end{array}\right| \\
& =(1,0.2)[\max \{\min (1,1), \min (1,1)\} \text {, } \\
& \min \{\max (0.4,0.5), \max (0.4,0.7)\}] \\
& +(1,0.4)[\max \{\min (1,1), \min (1,1)\} \text {, } \\
& \min \{\max (0.3,0.5), \max (0.4,0.5)\}] \\
& +(1,0.4)[\max \{\min (1,1), \min (1,1)\} \text {, } \\
& \min \{\max (0.3,0.7), \max (0.4,0.5)\}] \\
& =(1,0.2)(1,0.5)+(1,0.5)(1,0.4)+(1,0.3)(1,0.5)
\end{aligned}
$$


$=(1,0.5)+(1,0.5)+(1,0.5)$

$=(1,0.5)+(1,0.5)$

$=(1,0.5)$

$\operatorname{det} A^{c}=(1,0.5)$

Thus we arrive at the result

\subsubsection{Property4}

If $A^{c}$ and $B^{c}$ be fuzzy square matrices then

$$
\operatorname{adj}\left(A^{c} B^{c}\right)=\operatorname{adj} A^{c} \operatorname{adj} B^{c}
$$

Let us consider the fuzzy square matrix $B^{c}$ such that it is conformable for multiplication with previously cited fuzzy matrix $A^{c}$.

Let

$$
B^{c}=\left(\begin{array}{ccc}
(1,0.3) & (1,1) & (1,0.7) \\
(1,0.1) & (1,0.9) & (1,0) \\
(1,0.8) & (1,0.2) & (1,0.3)
\end{array}\right)
$$

Let us calculate the product

$A^{c} B^{c}$

$$
\begin{aligned}
& =\left(\begin{array}{ccc}
(1,0.5) & (1,0.3) & (1,0.8) \\
(1,0.6) & (1,0.2) & (1,0.9) \\
(1,0) & (1,0.7) & (1,0.4)
\end{array}\right) \\
& \left(\begin{array}{ccc}
(1,0.3) & (1,1) & (1,0.7) \\
(1,1) & (1,0.9) & (1,0) \\
(1,0.8) & (1,0.2) & (1,0.3)
\end{array}\right) \\
& =\left(\begin{array}{lll}
(1,0.5) & (1,0.8) & (1,0.3) \\
(1,0.6) & (1,0.9) & (1,0.2) \\
(1,0.3) & (1,0.4) & (1,0.4)
\end{array}\right)
\end{aligned}
$$$$
\operatorname{adj}\left(A^{c} B^{c}\right)=\left(\begin{array}{lll}
(1,0.4) & (1,0.3) & (1,0.6) \\
(1,0.4) & (1,0.3) & (1,0.5) \\
(1,0.8) & (1,0.5) & (1,0.8)
\end{array}\right)
$$

Then proceeding in the same manner we get

$$
\operatorname{adj} B^{c}=\left(\begin{array}{lll}
(1,0.2) & (1,0.7) & (1,0.9) \\
(1,0.8) & (1,0.3) & (1,0.3) \\
(1,0.9) & (1,0.2) & (1,0.9)
\end{array}\right)
$$

Thus we get

$\operatorname{adj} A^{c} a d j B^{c}$

$$
\begin{aligned}
& =\left(\begin{array}{lll}
(1,0.4) & (1,0.6) & (1,0.2) \\
(1,0.4) & (1,0.5) & (1,0.3) \\
(1,0.8) & (1,0.8) & (1,0.5)
\end{array}\right) \\
& =\left(\begin{array}{lll}
(1,0.2) & (1,0.8) & (1,0.9) \\
(1,0.7) & (1,0.3) & (1,0.2) \\
(1,0.9) & (1,0.3) & (1,0.9)
\end{array}\right) \\
& =\left(\begin{array}{lll}
(1,0.4) & (1,0.3) & (1,0.6) \\
(1,0.4) & (1,0.3) & (1,0.5) \\
(1,0.8) & (1,0.5) & (1,0.8)
\end{array}\right)
\end{aligned}
$$

Hence the result

$$
\operatorname{adj}\left(A^{c} B^{c}\right)=\operatorname{adj} A^{c} \operatorname{adj} B^{c}
$$

is verified.

\subsubsection{Property5}

If $A^{c}$ be fuzzy square matrices then

$$
\left(\operatorname{adj} A^{c}\right)^{\prime}=\operatorname{adj}\left(A^{c^{\prime}}\right)
$$

Proof:

$$
\operatorname{adj} A^{c}=\left[\left(1, b_{i j}\right)\right]
$$

Then

$$
\left(\operatorname{adj} A^{c}\right)^{\prime}=\left[\left(1, b_{j i}\right)\right]
$$

Again

$$
\operatorname{adj} A^{c^{\prime}}=\left[\left(1, b_{j i}\right)\right]
$$

In this way, we can claim that

$$
\left(\operatorname{adj} A^{c}\right)^{\prime}=\operatorname{adj}\left(A^{c^{\prime}}\right)
$$

To make the matter clear and simple, we have put forward an example in support of the claim.

\section{Numerical Example:}

We have

$$
\operatorname{adj} A^{c}=\left(\begin{array}{lll}
(1,0.4) & (1,0.6) & (1,0.2) \\
(1,0.4) & (1,0.5) & (1,0.3) \\
(1,0.8) & (1,0.8) & (1,0.5)
\end{array}\right)
$$

And so 


$$
\left(\operatorname{adj} A^{c}\right)^{\prime}=\left(\begin{array}{lll}
(1,0.4) & (1.0 .4) & (1,0.8) \\
(1,0.6) & (1,0.5) & (1,0.8) \\
(1,0.2) & (1,0.3) & (1,0.5)
\end{array}\right)
$$

Again

$$
\left(A^{c}\right)^{\prime}=\left(\begin{array}{ccc}
(1,0.5) & (1,0.6) & (1,0) \\
(1,0.3) & (1,0.2) & (1,0.7) \\
(1,0.8) & (1,0.9) & (1,0.4)
\end{array}\right)
$$

And

$$
\left(\operatorname{adj} A^{c}\right)^{\prime}=\left(\begin{array}{lll}
(1,0.4) & (1,0.4) & (1,0.8) \\
(1,0.6) & (1,0.5) & (1,0.8) \\
(1,0.2) & (1,0.3) & (1,0.5)
\end{array}\right)
$$

Hence we get

$$
\operatorname{adj}\left(A^{c}\right)^{\prime}=\left(\operatorname{adj} A^{c}\right)^{\prime}
$$

These are some of the properties which are found to hold for the determinant of square fuzzy matrices represented with the help of reference function.

\section{Conclusions}

In this article, our main intention was to represent fuzzy matrices according to reference function and then to define the determinant and adjoint of square fuzzy matrices accordingly. We thin $\mathrm{k}$ that the representation of fuzzy sets with the help of reference function is very essential to have logical frameworks. Again while defining the determinant and adjoint of square fuzzy matrices with the help of reference function, some of the properties of the determinant as well as the adjoint of square fuzzy matrices were studied. In the process, it is found that the properties of the determinat and adjoint of square fuzzy matrices are somewhere analogus with the crisp cases.

\section{References}

[1] S.V Ovehinnikov, Structure of fuzzy relations, Fuzzy Sets and Systems, 6(1981), 169-195.

[2] M.G Thomson, Convergence of powers of a fuzzy matrix, J. Math. Anal. Appl. 57, 476-480, Elsevier, 1977.

[3] H Hasimato, Convergence of powers of fuzzy transitive matrix, Fuzzy Sets and Systems, 9(1983), $153-160$

[4] A Kandel, Fuzy Mathematical Techniques with Applications, Addition Wisley, Tokyo, 1996.
[5] W Kolodziejezyk, Convergence of s-transitive fuzzy matrices, Fuzzy Sets and System, 26(1988), 127-130.

[6] J.B Kim, A. Baartmans Determinant Theory for Fuzzy Matrices, Fuzzy Sets and Systems, 29(1989), 349-356.

[7] J.B Kim, Determinant theory for Fuzzy and Boolean Matices, Congressus Numerantium Utilitus Mathematica Pub (1978), 273-276.

[8] J.B Kim, Idempotents and Inverses in Fuzzy Matrices, Malayasian Math 6(2)1988, Management Science.

[9] J.B Kim, Inverses of Boolean Matrices, Bull.Inst. Math. Acod, Science 12(2)(1984), 125-128

[10] Baruah H K, Fuzzy Membership with respect to a Reference Function, Journal of the Assam Science Society, 1999, 40(.3):65-73.

[11] Baruah H K, Towards Forming A Field of Fuzzy Sets, International Journal of Energy Information and Communications, 2011, 2(1): $16-20$.

[12] Baruah H K, Theory of Fuzzy sets Beliefs and Realities, International Journal of Energy, Information and Communications, 2011, 2(2): 1-22

[13] Dhar M, On Hwang and Yang's definition of Entropy of Fuzzy sets, International Journal of Latest Trend Computing, 2011, 2(4): 496-497.

[14] Dhar M, A Note on existing Definition of Fuzzy Entropy, International Journal of Energy Information and Communications, 2012, 3( 1): 1721.

[15] Dhar M, On Separation Index of Fuzzy Sets, International Journal of Mathematical Archives, 2012, .3(3): 932-934.

[16] Dhar M, On Geometrical Representation of Fuzzy Numbers, International Journal of Energy Information and Communications, 2012, 3(2): 2934.

[17] Dhar M, On Fuzzy Measures of Symmetry Breaking of Conditions, Similarity and Comparisons: Non Statistical Information for the Single Patient., Accepted for publication in International Journal of Mathematical Archives, 2012.

[18] Dhar M, A Note on Subsethood measure of fuzzy sets, accepted for publication in International Journal of Energy, Information and Communications, 2012.

\section{Author's Profiles}

Mamoni Dhar is an Assistant Professor in the department of Mathematics, Science College, Kokrajhar-783370, Assam, India. She received M.Sc 
degree from Gauhati University, M.Phil degree from Madurai Kamraj University, B.Ed from Gauhati University and PGDIM from Indira Gandhi National Open University. She had received National Scholarship from Matriculation to Post Graduation. Her research interest is in Fuzzy Set Theory from new perspectives. She has published eleven articles in different national and international journals.

How to cite this paper: Mamoni Dhar,"A Note on Determinant and Adjoint of Fuzzy Square Matrix", International Journal of Intelligent Systems and Applications(IJISA), vol.5, no.5, pp.58-67, 2013.DOI: 10.5815/ijisa.2013.05.07 